before viral encounter, would produce many fewer virus-resistant bacteria.

On the basis of this insight, Luria and Delbrück generated a statistical distribution (the Luria-Delbrück distribution) to describe the prevalence of virus-resistant bacterial mutants that would be expected if mutations arose randomly before the bacterial population came under selective pressure from the virus. Compared with a Poisson distribution expected for adaptive mutations, this LuriaDelbrück distribution has a long 'tail' at the end of the distribution pattern. In the context of the authors' experiments, this tail would correspond to Petri dishes that have a high number of bacterial colonies, corresponding to early mutational events that lead to a large number of mutant descendants.

The 1943 paper reported the results of the authors' experiments, termed fluctuation tests, that took this mathematical approach to analyse the number of virus-resistant colonies in E. coli populations. The authors' findings were consistent with mutations following a Luria-Delbrück distribution rather than a Poisson distribution, demonstrating that bacterial mutations arose randomly, and independently of an encounter with a virus.

Luria and Delbrück's work shaped subsequent studies of biology and evolution in many ways. Luria himself was reported as saying that their fluctuation test removed bacteria from "the last stronghold of Lamarckism" (see go.nature.com/2fbxujf). The fluctuation test is still a standard procedure for accurately measuring mutation rates in diverse systems, from bacteria ${ }^{8}$ and yeast ${ }^{9}$ to cancer cells $^{10}$. Their study also popularized the use of E. coli and the viruses that attack it as a simple experimental model system for biology ${ }^{11}$. Beyond its direct impact in laboratories, the experiment became a textbook example of how mathematical thinking combined with simple experimentation can lead to profound biological insights $^{12}$. For their contributions to bacterial and viral genetics, Luria and Delbrück won the Nobel Prize in Physiology or Medicine in 1969 (which they shared with the biologist Alfred Hershey).

Their insight into mutational processes also has implications in settings such as the clinic. In analogy to the original experiment, imagine a population of patients who have the same type of bacterial infection and who are being treated with the same antibiotic (the antibiotic replaces the virus as the selection pressure here). According to the randommutation model, even if all else is equal among the patients, the number of antibioticresistant bacterial mutants initially present will vary highly between the patients, which could lead to markedly variable treatment outcomes. Because such high inherent variability in treatment efficiency reflects resistance mutations arising in a population before treatment, using DNA sequencing or other types of analysis to identify the presence and number of antibiotic-resistant bacterial mutants before treatment could improve our ability to predict treatment outcome.

Did the Luria and Delbrück study really close the door on Lamarckism? As far as bacteria are concerned, the answer is much more complicated than the duo could probably ever have anticipated.

It is undeniable today that randomly occurring mutations and natural selection are central tenets of how bacterial evolution occurs ${ }^{5}$. However, scientists are uncovering and debating an increasing array of other evolutionary processes at work in bacteria, some of which are suspiciously Lamarckian in character ${ }^{4-6}$. For example, we now know that the genome-wide mutation rate, and even the mutation rates of specific genes, can be shaped by evolution and affected by the environment ${ }^{13-15}$. An even more striking example is bacterial adaptation through the CRISPR-Cas viral-defence system, in which bacteria can incorporate viral genetic material into their own genomes and use it, as an adaptive mechanism, to protect themselves and their descendants against current and subsequent viral attacks ${ }^{16,17}$. These quasi-Lamarkian mechanisms presumably evolved by random mutations and natural selection. They do not necessarily undermine the lessons learnt from Luria and Delbrück's work, but rather, show the power of evolution to sculpt living organisms in endlessly interesting ways.

It is intriguing to imagine an alternative scientific history that might have occurred if Luria and Delbrück had stumbled upon one of these quasi-Lamarkian mechanisms. The CRISPR-Cas defence mechanism is mainly repressed in the E. coli that they studied, but it is active in other bacterial species, such as Streptococcus thermophilus.
A fun challenge would be to repeat the Luria-Delbrück experiment under conditions that might favour the evolution of resistance by such adaptive mechanisms, for example by replacing E. coli with $S$. thermophilus. Would the distribution of the number of resistant mutants indicate random or adaptive mutations? What would Luria and Delbrück have concluded had they used a species that had the CRISPR-Cas system? The contingency of this historic choice underscores the fact that, like evolution, science perhaps also progresses both adaptively and randomly.

Manoshi S. Datta and Roy Kishony are in the Faculty of Biology, Technion-Israel Institute of Technology, Haifa 3200003, Israel. e-mails:mdatta@campus.technion.ac.il; rkishony@technion.ac.il

1. Luria, S. E. \& Delbrück, M. Genetics 28, 491-511 (1943).

2. Zheng, Q. Chance 23 (2), 15-18 (2010).

3. Lenski, R. E. PLoS Genet. 13, e1006668 (2017).

4. Koonin, E. V. \& Wolf, Y. I. Biol. Direct 4, 42 (2009).

5. Sniegowski, P. D. \& Lenski, R. E. Annu. Rev. Ecol. Syst. 26, 553-578 (1995).

6. Rosenberg, S. M. Nature Rev. Genet. 2, 504-515 (2001).

7. Luria, S. E. A Slot Machine, a Broken Test Tube: An Autobiography (Harper \& Row, 1984).

8. Bell, G. \& MacLean, C. Trends Microbiol. 26, 471-483 (2018)

9. Farlow, A. et al. Genetics 201, 737-744 (2015).

10.Powers, S. \& Pollack, R. E. Nature Rev. Cancer 16, 266-270 (2016)

11.Cairns, J., Stent, G. S. \& Watson, J. D. Phage and the Origins of Molecular Biology (Cold Spring Harb. Press, 2007).

12. Murray, A. Genetics 202, 367-368 (2016).

13. Tenaillon, O., Denamur, E. \& Matic, I. Trends Microbiol. 12, 264-270 (2004).

14. Moxon, E. R., Rainey, P. B., Nowak, M. A \& Lenski, R. E. Curr. Biol. 4, 24-33 (1994).

15.Wielgoss, S. et al. Proc. Natl Acad. Sci. USA 110, 222-227 (2013).

16. Marraffini, L. A. Nature 526, 55-61 (2015).

17.Koonin, E. V. \& Wolf, Y. I. Biol. Direct 11, 9 (2016).

\title{
The not-so-dangerous lives of Neanderthals
}

Have Neanderthals gained an unfair reputation for having led highly violent lives? A comparison of skulls of Neanderthals and prehistoric humans in Eurasia reveals no evidence of higher levels of trauma in these hominins. SEE LETTER P.686

\section{MARTA MIRAZÓN LAHR}

$\mathrm{I}$ njuries are part of everyday life, from a scratch on the skin to a broken bone to a fatal trauma. Although many injuries are accidental, others can arise as a consequence of an individual's or a group's behaviour, activity or social norms - characteristics that tell us about societies and the inherent tensions and risks within and between different groups.
On page 686 , Beier et al. ${ }^{1}$ provide evidence that challenges the long-standing view ${ }^{2}$ that Neanderthal populations experienced a level of traumatic injuries that was significantly higher than that of humans. The result calls into question claims ${ }^{2,3}$ that the behaviour and technologies of Neanderthals exposed them to particularly high levels of risk and danger.

Reports of injuries and deaths are constantly in the news. As well as being drawn to read the 
stories of individuals, such information is of interest because of what it tells us about our societies. However, to fully understand what might determine the current degree of violence and injuries, we also need to look back at the past and identify the causal underpinnings. But how far back should we look? Arguably, right back to the evolutionary origins of processes that shape behavioural, social and cognitive tendencies and abilities.

Anthropologists study skeletal remains to reconstruct aspects of ancient lives, building an 'osteobiography' that casts light on part of the life history of an individual. Skeletons preserve - in the form of holes, misshapen surfaces, bone misalignments and secondary fractures radiating out from a point of impact - a signature of the traumas that resulted in fractured, cut or perforated bones, even if the injuries subsequently healed ${ }^{4,5}$.

Traumatic lesions have been frequently identified in Neanderthal fossils, particularly in the head (Fig. 1) and neck, leading to the view ${ }^{2}$ that higher levels of skeletal injury occurred in Neanderthal populations than in human populations. However, this is not so, say Beier and colleagues. The authors assessed published descriptions of Neanderthal and modern human fossil skulls found in Eurasia from approximately 80,000 to 20,000 years ago. Comparing the number of injured and noninjured Neanderthal and human skulls, the authors report similar levels of head trauma in both groups.

The power of Beier and colleagues' analyses lies in their study design. Instead of comparing Neanderthal data with those of more-recent or living human populations, as previous studies have done $\mathrm{e}^{2,6}$, the authors based their comparisons on humans who not only shared aspects of their environment with Neanderthals, but whose fossil record also has a similar level of preservation. Beier et al. analysed data for 114 Neanderthal skulls and 90 human skulls. They gathered the data for 14 skull bones, and obtained information that ranged from 1 bone in poorly preserved fossils to data for all 14 bones per individual for wellpreserved ones. In total, the authors recorded trauma incidence in 295 Neanderthal bones and 541 human bones. They also collected other information, such as the percentage of each of the 14 bones that was preserved for each individual, as well as details including sex, age at death and the fossil's geographic location.

Beier et al. ran two sets of statistical analyses - one based on the presence or absence of trauma in each of the skull bones, the other on individual fossil skulls as a whole - to test whether there were any statistically significant differences between the prevalence of trauma in the Neanderthal and human fossils. The authors also assessed whether trauma prevalence was linked to sex or age, taking into account fossil preservation, geographic location and possible interaction effects between the different variables.

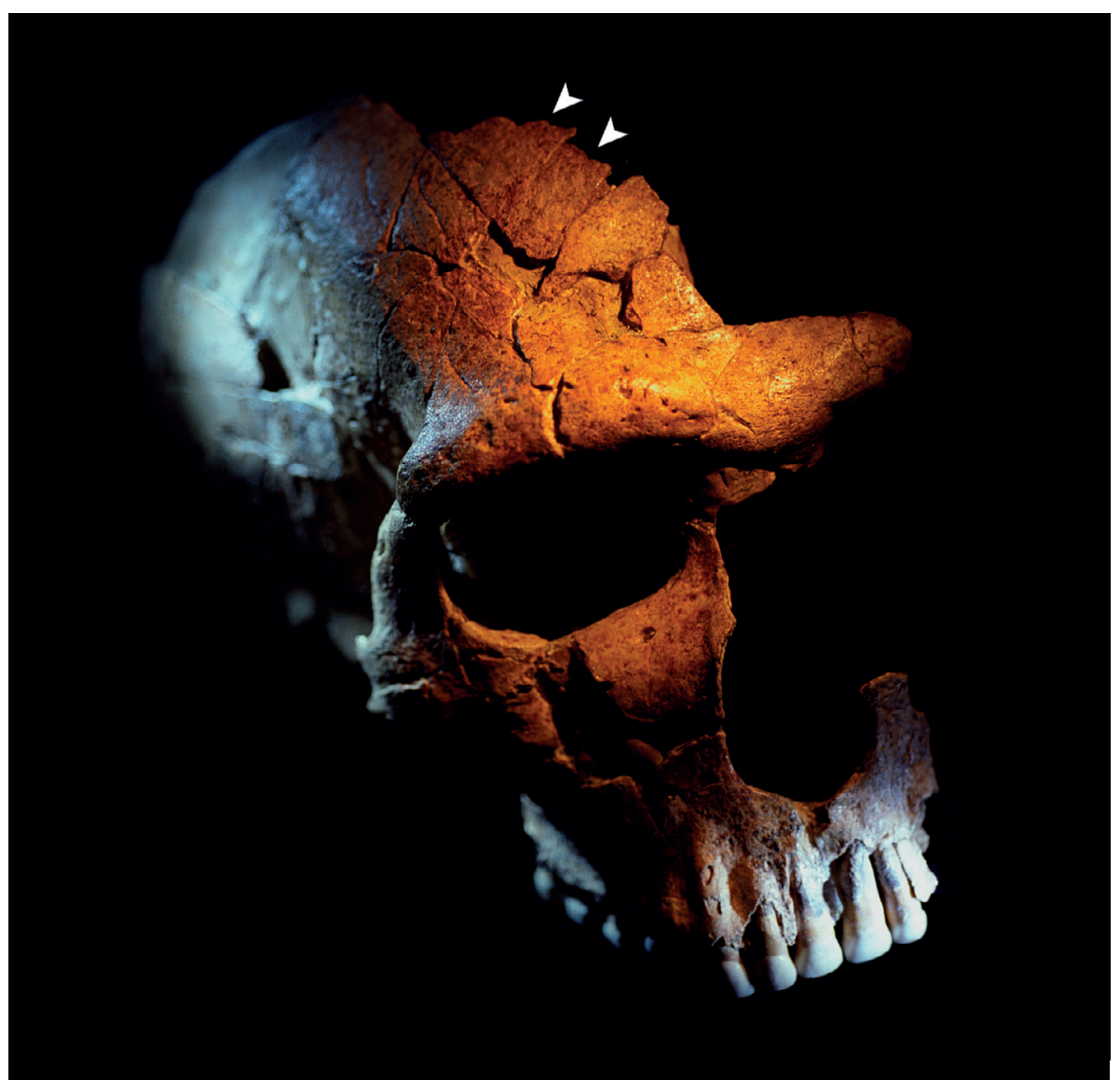

Figure 1 | A Neanderthal skull. The Neanderthal fossil called Saint-Césaire 1. This fossil ${ }^{9}$ shows signs of a healed bone injury ${ }^{10}$ in the region indicated by the arrowheads. Beier et al. ${ }^{1}$ assessed published analyses of ancient Neanderthal and human skulls, including that of Saint-Césaire 1. Contrary to the prevailing view $^{2}$ that Neanderthal existence was more violent than that of humans, the authors report that similar levels of trauma are present in Neanderthal and human fossils.

The two analyses gave similar results.

The more complete the fossils are, the more likely they are to have preserved evidence of injuries. This might seem obvious, but is an issue often ignored in such studies. Beier et al. offer a way to deal with this type of bias in the available material. Once the authors take into account the extent of fossil preservation, the predicted prevalence of trauma in

\section{"Risk and danger were as much a part of the life of Neanderthals as they were of our own evolutionary past."} Neanderthals and humans is almost the same.

Both Neanderthal and human males had a much greater incidence of trauma than did the females of their respective species. This pattern remains the same for humans today ${ }^{7}$. One final intriguing result is that, although traumatic injuries were present across all of the age ranges studied, Neanderthals that had trauma to the head were more likely to have died under the age of 30 than the humans were. The authors interpret this result as evidence that, compared with humans, Neanderthals either had more injuries when they were young or were more likely to have died after being injured.

Beier and colleagues' study does not invalidate previous estimates of trauma among Neanderthals. Instead, it provides a new framework for interpreting these data by showing that the level of Neanderthal trauma was not uniquely high relative to that of early humans in Eurasia. This implies that Neanderthal trauma does not require its own special explanations, and that risk and danger were as much a part of the life of Neanderthals as they were of our own evolutionary past. The result adds to growing evidence that Neanderthals had much in common with early human groups. However, the finding that Neanderthals might have experienced trauma at a younger age than humans, or that they had a greater risk of death after injury, is fascinating, and might be a key insight into why our species had such a demographic advantage over Neanderthals.

Is this the final word on the subject of Neanderthal trauma? The answer is no. Beier and colleagues assessed only skull trauma. What if Neanderthals accumulated more injuries to their bodies than did humans? There are data suggesting that this might be the case ${ }^{8}$. Furthermore, although the authors' analyses 
demonstrate the power of a well-designed study based on large samples, the data they used were recorded by many researchers and at varying levels of detail, raising the possibility of methodological biases.

Lastly, the causes of the injuries could provide some elusive insights into behaviour, activities or social norms in the past. From the shape, location and extent of traumatic injuries in skeletons, and characteristics such as the sharpness of fracture edges or the degree to which injuries had healed, it is sometimes possible to establish the most likely cause of a trauma - for example, whether the injury probably arose as a consequence of a hunting accident $^{3}$, interpersonal violence ${ }^{10}$ or intergroup conflict ${ }^{11}$. Moreover, surviving severe trauma might indicate that the injured person was cared for by members of their society ${ }^{12}$. Establishing the likelihood of each of these scenarios among Neanderthals and early modern humans will no doubt continue to challenge scientists for many years to come.

Marta Mirazón Lahr is in the Department of Archaeology, Leverhulme Centre for Human Evolutionary Studies, University of Cambridge, Cambridge CB2 1QH, UK. e-mail:mbml1@cam.ac.uk

1. Beier, J., Anthes, N., Wahl, J. \& Harvati, K. Nature 563, 686-690 (2018).

2. Berger, T. D. \& Trinkaus, E. J. Archaeol. Sci. 22 841-852 (1995).

3. Churchill, S. E. Evol. Anthropol. 11, 185-186 (2002).
4. Redfern, R. C. Injury and Trauma in Bioarchaeology. Interpreting Violence in Past Lives (Cambridge Univ. Press, 2017)

5. Lovell, N. C. in Biological Anthropology of the Human Skeleton (eds Katzenberg, M. A. \& Saunders, S. R.) 341-386 (Wiley, 2008)

6. Underdown, S. J. Period. Biol. 108, 485-493 (2006).

7. Pinker, S. The Better Angels of Our Nature: A History of Violence and Humanity (Penguin, 2011).

8. Trinkaus, E. J. Archaeol. Sci. 39, 3691-3693 (2012).

9. Lévêque, F. \& Vandermeersch, B. C. R. Acad. Sci. 291, 187-189 (1980)

10.Zollikofer, C. P. E., Ponce de Léon, M. S., Vandermeersch, B. \& Lévêque, F. Proc. Natl Acad. Sci. USA 99, 6444-6448 (2002).

11. Mirazón Lahr, M. et al. Nature 529, 394-398 2016).

12.Spikins, P., Needham, A., Tilley L. \& Hitchens, G. E. World Arch. https://doi.org/10.1080/00438243.20 18.1433060 (2018).

This article was published online on 14 November 2018.

\section{A glimpse into the heart of a quasar}

of electromagnetic radiation coming from a single point to infer the structure and dynamics of the gas and dust around the black hole. Such properties include colour, time variability, polarization and phase - the offset of an electromagnetic wave from a given position.

For the past 30 years, our best understanding of gas in the vicinity of a quasar's black hole has come from a method called reverberation mapping, which uses echoes of light (analogous to those of sound) to map out regions near the black hole ${ }^{5}$. The accretion disk emits light in all directions, some of which is observed directly by telescopes, and some of which illuminates a region of surrounding gas, known to astronomers as the broad-line region. Optical-reverberation mapping measures how long it takes the broad-line region
W hen the astronomical object 3C 273 was detected $^{1}$, to most optical telescopes it looked just like a star in our Galaxy. But in 1963, astronomers discovered $^{2,3}$ that the object was shining from a distance of 750 megaparsecs (2.4 billion light years). Whatever this mystery object was, it was producing more radiation than a trillion stars, from a region no bigger than the Solar System. Objects such as 3C 273 are now known as quasars and are understood to be powered by hot gas and dust feeding into a supermassive black hole through a structure called an accretion disk. Fifty-five years after that remarkable discovery, 3C 273 is back in the limelight. On page 657, the GRAVITY Collaboration ${ }^{4}$ reports observations of the spatially resolved rotation of hot gas in the quasar at distances much closer to the black hole than were previously possible.

A quasar can produce more energy than the entire galaxy in which it resides. Although the basic mechanism that powers a quasar is known, the anatomy of the supermassive black hole and its surroundings is not well understood. Where does the gas that feeds the black hole come from? And what effect does the resulting intense radiation have on the environment around the black hole? The findings of the GRAVITY Collaboration provide a way to answer these fundamental questions.

Determining the structure of a quasar is difficult because the black hole is extremely small and far away from Earth, and therefore the gas orbiting close to the black hole cannot be directly imaged using telescopes. Instead, astronomers rely on the properties

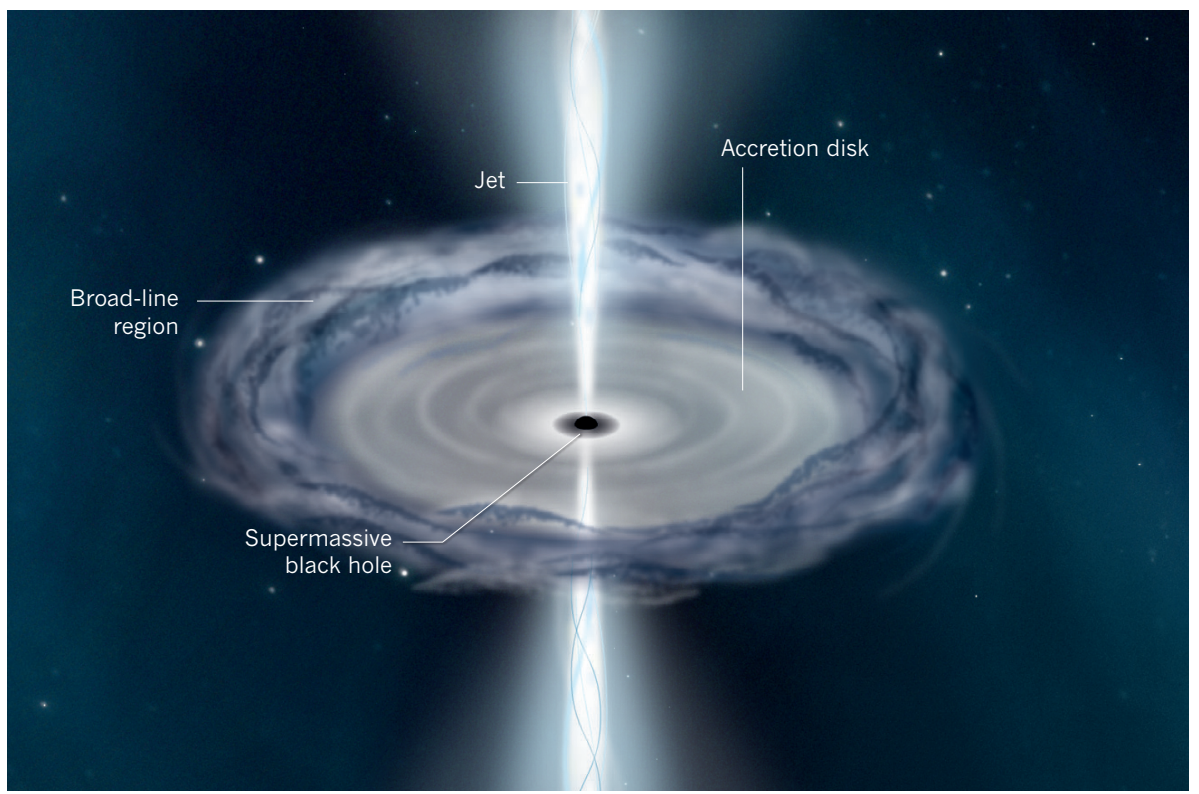

Figure 1 | Structure of the quasar 3C 273. Quasars are astronomical objects comprising a supermassive black hole surrounded by hot gas and dust. As this material is pulled towards the black hole through a structure known as an accretion disk, energy is released in the form of light and, in the case of the quasar 3C 273, as a beam of charged particles called a jet. The GRAVITY Collaboration ${ }^{4}$ reports a technique that enables the rotation of gas in a part of $3 \mathrm{C} 273 \mathrm{known}$ as the broad-line region to be spatially resolved. The researchers determine that this gas moves perpendicular to the jet and has the shape of a thick ring. 